\title{
Directional and nondirectional spectral reflection from the human fovea
}

\author{
Jan van de Kraats \\ Dirk van Norren \\ University Medical Center Utrecht \\ Department of Ophthalmology AZU E03.136 \\ P.O. Box 85500 \\ NL-3508 GA, Utrecht, The Netherlands
}

\begin{abstract}
A model of the directional and nondirectional reflection spectrum of the human fovea is developed, incorporating reflectors, absorbers, and a wavelength-dependent optical Stiles-Crawford effect (OSCE). Data from 102 healthy subjects between 18 and 75 years obtained with the fundus reflection analyzer (FRA), an imaging spectrograph that measures the directional reflection profile of the human fovea in the pupil plane from 400 to $950 \mathrm{~nm}$, were analyzed. Subgroups of young ( $<40$ years) and old ( $>50$ years) observers were defined. Mean results of the young group defined a template for directionality versus wavelength. For the whole group, mean reflection at $550 \mathrm{~nm}$ from the cones was $2.12 \%$, from the retinal pigment epithelium $0.56 \%$, and from the choroid $7.92 \%$. Lens density, cone disc reflection, and blood layer thickness showed significant trends versus age. The model for the first time simultaneously describes the spectra of the directional and nondirectional reflection of the human fovea. Rayleigh scatter losses of the media and in preretinal layers were assumed zero in the nondirectional pathway. Mean density of the macular pigment of a subgroup (53 subjects, 19 to 75 years) correlated significantly with independent data from reflectance and autoflourescence images obtained by scanning laser ophthalmoscope (SLO) and data from flicker photometry. $\odot 2008$ Society of Photo-Optical Instrumentation Engineers. [DOI: 10.1117/1.2899151]
\end{abstract}

Keywords: optical Stiles-Crawford effect (OSCE); cone receptor reflectance; macular pigment; melanin; spectroscopy; eye media optical density.

Paper 07213RR received Jun. 13, 2007; revised manuscript received Nov. 5, 2007; accepted for publication Nov. 5, 2007; published online Mar. 25, 2008.

\section{Introduction}

Several attempts have been made to model the spectral reflection of the fundus of the eye by assuming reflection at different retinal layers interspaced with ocular absorbers. In 1986, Van Norren and Tiemeijer ${ }^{1}$ measured the reflectance of the fovea, a peripheral site, and the optic disc at 14 wavelengths. Measurements were rather cumbersome, as each wavelength needed a separate session. Their instrument optimized the directional reflection from the receptors (the optical StilesCrawford effect, or OSCE) by using entry and exit pupils that were small and close together. Their model had two reflecting layers, one at the retinal pigment epithelium (RPE) and one at the sclera. In addition, four absorbers were distinguished; lens, macular pigment, melanin, and blood. In that same year, Van Blokland and Van Norren ${ }^{2}$ presented reflectance measurements with an instrument that enabled detailed information about the shape of the directional reflection, but only four laser wavelengths were available. In a descriptive model, the authors suggested several trajectories that light takes in the receptor layer.

Address all correspondence to Jan van de Kraats, UMC Utrecht, Department of Ophthalmology, AZU E03.136, P.O. Box 85500, NL-3508 GA Utrecht, The Netherlands; Tel: +31 30 2506775; Fax: +31 30 2505417; E-mail: jkraats@umcutrecht.nl
A few years later, Delori and Pflibsen ${ }^{3}$ build a reflectometer based on a grating spectrometer, allowing all wavelengths to be measured simultaneously. The optical design used the exit pupil of a Zeiss fundus camera and thus was not optimized for obtaining the directional reflection from the receptors. Their model added a graded reflection from the choroidal space to the model of Van Norren and Tiemeijer. ${ }^{1}$ They achieved a better estimate of absorption in melanin and blood, but the receptor layer was ignored. Van Norren and Van de Kraats presented an instrument that could measure 15 wavelengths quasi-simultaneously. ${ }^{4}$ The instrument was again optimized for the directional reflection, and it used low light levels to enable retinal reflection spectra with the nearly full complement of visual pigments. A new model was derived by Van de Kraats et al. with better funded trajectories of light in the receptor layer. ${ }^{5}$ Spectral reflection was measured at two pupillary positions, one at the maximum of the directional reflection and one at a position with the entry and exit pupils shifted horizontally by $2.5 \mathrm{~mm}$. A reflection from the stack of cone receptor discs was incorporated in the model, based on measurements with high and low levels of visual pigment and with high and low directional reflection. The model permitted the isolation of pre-receptor, receptor, and post-receptor signals, thereby improving the accuracy of estimating the pre-

$1083-3668 / 2008 / 13(2) / 024010 / 13 / \$ 25.00$ C 2008 SPIE 
receptor parameters and lens and macular pigment density.

A new instrument proposed by Van de Kraats and elaborated by Zagers et al. ${ }^{6}$ combined the directional resolution earlier presented by Van Blokland and Van Norren, ${ }^{2}$ with the spectral resolution of Delori and Pflibsen. ${ }^{3}$ In 1 second, a two-dimensional (2-D) image could be measured from a foveal spot illuminated with intense white light, containing in one dimension the spectral aspects and in the other the directional aspects. The fast measurement allows large samples. In their data analysis, Zagers et al., ${ }^{6}$ as a first approach, used the directional component of the signal to estimate the prereceptor absorbers and parameters for the optical quality of the receptor layer.

The purpose of the present study was to propose and test a model that fully explains both the spectral and the directional aspects of the reflection of the human fovea, i.e., the directional cone reflection and the nondirectional background reflection. The model generates quantitative information about reflectors and absorbers. In addition, the dependence on age of these parameters was studied. A validation of the estimates of macular pigment density was obtained with three other methods in an identical subgroup of 53 subjects.

\section{Theory}

\subsection{Optical Stiles-Crawford Effect in the Pupil Plane}

The angular reflection, $R_{(x)}$, from an illuminated spot at the fovea shows up in the pupil plane as a Gaussian-shaped spatial intensity distribution (the directional component with amplitude $A$ ) on a pedestal of background light (the nondirectional component $B):^{2,7,8}$

$$
R(x)=B+A \times 10^{-\rho\left(x-x_{0}\right)^{2}},
$$

with $\rho$ the directionality, and $x$ the horizontal location in $\mathrm{mm}$ in the pupil plane relative to the center of the pupil. The peak of the OSCE is generally not exactly at the center of the pupil; the difference is $x_{0}$. Measured curves are cut off by the edges of the (generally dilated) pupil (Fig. 1).

\subsection{Spectral Behavior of Directionality $\rho$}

The directionality $\rho$ of the Gaussian varies with wavelength $^{9-11}$ :

$$
\rho(\lambda)=\rho_{w g}+\rho_{\text {scatt }} \times(550 / \lambda)^{2},
$$

with $\rho_{w g}$ a waveguide component inherent to the receptors that is constant over wavelength, $\rho_{\text {scatt }}$ a scalar preceding a scatter component that varies with wavelength due to interference effects between receptors, and $\lambda$ the wavelength in $\mathrm{nm}$ (Fig. 2; the data are described in Sec. 5). The equation stems from Marcos et al., ${ }^{9}$ but it was slightly modified by the normalization at $550 \mathrm{~nm}$ to enable easy comparison of the contributions of $\rho_{w g}$ and $\rho_{\text {scatt }}$.

\subsection{Origin of the Directional Component}

The origin of the directional reflection has been located on the discs of the cone receptors ${ }^{5}$ or at the end of the outer segments. $^{12}$ Experiments with the visual pigments in a bleached condition as used throughout this paper cannot discriminate between these two options. In line with our earlier

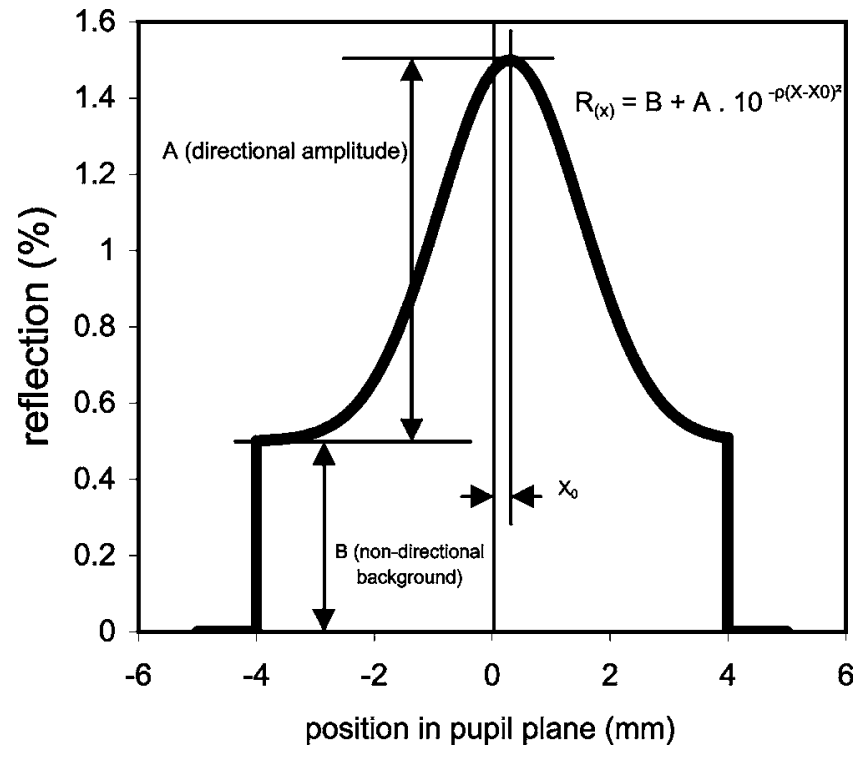

Fig. 1 Directional and nondirectional light measured across the (8$\mathrm{mm}$ ) pupil of the eye (artificial data). The directional part, the OSCE, has a Gaussian shape. It sits on a pedestal of nondirectional background light.

interpretation ${ }^{5}$ based on measurements in both the dark and the bleached states of the visual pigments, we opted for choosing the discs as the source of the directional reflection. The reflection from the layers posterior to the receptor layer is considered nondirectional. The principle is illustrated in Fig. 3 , where ray tracing through a lens and through a more complex refractive element, a receptor, results in the same reflection value as from the bare surface. In the retina, finally all rays through the receptor layer, guided or nonguided, hit the layers posterior to it, including the highly scattering choroid. Reciprocity theory states that the reflected rays take the same paths on their way back, preserving the wide-angle scattering nature, at least over the full width of the pupil.

To prove this, Van de Kraats et al. ${ }^{5}$ described an experiment with an annulus around the measuring area to illuminate the receptor layer only from the choroidal side. As expected, the reflected light from the central, nonilluminated part showed heavy absorption because of blood and melanin, but no directionality. Similarly, in experiments from the Burns

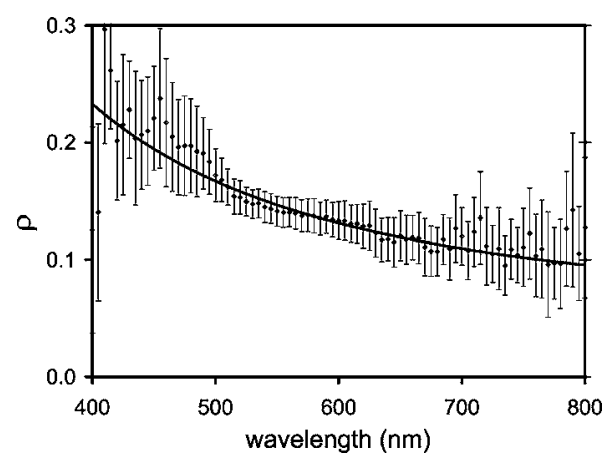

Fig. 2 The directionality $\rho$ as a function of wavelength. Data points are the mean of the young group (see Sec. 5.2); error bars are the mean standard errors per subject. 


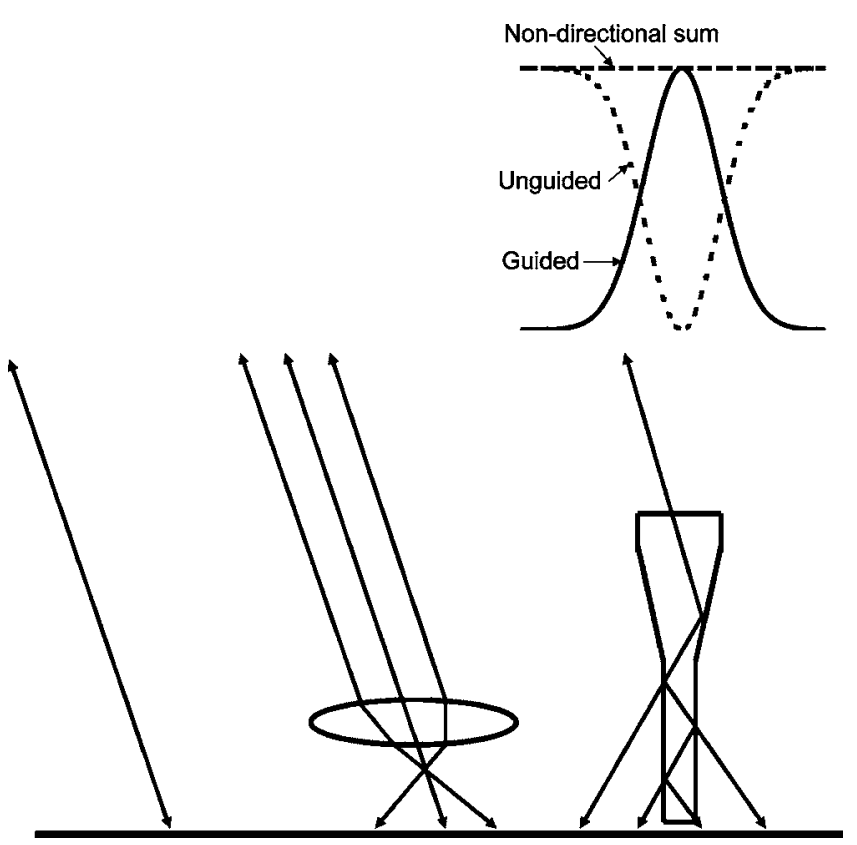

Fig. 3 Different refractive elements in front of a diffusely scattering Lambertian surface, all leading to nondirectional behavior. At the left, a measuring device is looking directly at the surface (only one angle shown). At the center, light is bent through a lens. At the right, light is guided through and escapes from a bleached cone receptor. The pathways to the surface are similar on the way back. Above the cone receptor, the intensity distribution at some distance (pupil plane in the eye) is shown, with the fraction guided through the receptor, the unguided fraction escaping the receptor, and the sum of both, forming again a nondirectional background.

group using the fluorescence of lipofuscin in the pigment epithelium as a new source of light located behind the receptors, no directional light was found. ${ }^{12-15}$ The implication of both types of experiment is not that light avoids the pathway through the photoreceptors on its way back, ${ }^{12}$ but rather that the Gaussian-shaped directional light guided through (recaptured by) the photoreceptor outer segments is perfectly complemented with inverted Gaussian-shaped light through the pathways passing the outer segment wall. These pathways are in reversal to that of the (nonperpendicular) light escaping the outer segments on entering the cones from the direction of the pupil (Fig. 3). In conclusion, any directional light must originate from reflections in the cones themselves (including the outer segment tips), and not from light that also traveled the layers behind it.

\subsection{Cone Capture Area}

From (optical) antenna theory, ${ }^{16}$ it follows that the capture area of a cone is not purely determined by its physical dimensions, but can be larger. ${ }^{5,17}$ Estimations using waveguide theory also give a significantly higher effective capture area compared to the physical frontal area of a cone. ${ }^{18}$ We therefore assumed that all perpendicular light enters the cones and that none reaches the interspaces. For oblique angles, light escapes from the cone outer segment into the interspaces, according to the generally accepted explanation of the StilesCrawford effect. ${ }^{19}$ Energy distributions calculated in rods showed almost identical behavior for wavelengths of 475 ,
505 , and $714 \mathrm{~nm} .{ }^{20}$ On this basis, we also assumed that, again for the perpendicular case, the light captured by the foveal cones is constant over wavelength. In the bleached condition, the light passes the cone outer segments into the deeper layers. Due to the reciprocity principle, a large part of the light reflected from the deeper layers must be recaptured by the cones.

\section{Model}

\subsection{Overview}

An earlier version of the model with an extensive discussion was published by Van de Kraats et al. ${ }^{5}$ The main deviations in the present model concern the regaining of scattered light of the nondirectional component, a wavelength-dependent reflection from the cones, the use of a tapered blood layer thickness, and new templates for the eye media and the macular pigment. A schematic view of the model is given in Fig. 4. Briefly, with the directional reflection originating from the cone receptors, only absorbers anterior to the cones, i.e., lens and macular pigment, leave their spectral fingerprint. ${ }^{5,21}$ The nondirectional amplitude is in addition influenced by absorption in blood and melanin. ${ }^{3}$ In the anterior eye, light is absorbed by the media. The first (nondirectional) reflection occurs in front of the receptor layer, at the internal limiting membrane (ILM). Although the reflection at the ILM is specular and therefore directional, it does illuminate the pupil quasi-uniformly and can therefore be treated as nondirectional because it emanates from an image of the source located very close to the retina. ${ }^{22}$ Next, the only directional reflection takes place at the cone outer segments. A further nondirectional reflection occurs at the retinal pigment epithelium. Last, light is reflected at the choroid, with absorption in blood and melanin. To avoid complex formulas, losses at reflecting layers of a few percent were ignored in the calculation of posterior layers. Visual pigments were supposed to be fully bleached.

\subsection{Absorbers in Both Directional and Nondirectional Components}

The transmission of the various absorbing layers was described by their optical density (Fig. 5). In general,

$$
T=10^{- \text {dens }} .
$$

The density of the media is given in our recent model $^{23}$

$$
\begin{aligned}
D_{\text {meddir }}(\lambda)= & d_{R L} \times M_{R L}(\lambda)+d_{L Y} \times M_{L Y}(\lambda)+d_{L O} \times M_{L O}(\lambda) \\
& +24 \times M_{\text {water }}(\lambda) .
\end{aligned}
$$

Briefly, $M_{R L}(\lambda)$ is a spectral template for losses in Rayleigh scatter in the cornea, humors, and lens; $M_{L Y}(\lambda)$ is a template for losses in the young lens; and $M_{L O}(\lambda)$ is for losses in the aging lens. The parameters $d_{R L}, d_{L Y}$, and $d_{L O}$, derived from fitting the measured data to the model, were the agedependent densities at $400 \mathrm{~nm}$. A term $d_{\text {neutral }}$ for a neutral density of the media to provide for some losses due to scatter by relatively large scattering particles was ignored. $M_{\text {water }}(\lambda)$ is the density of $1 \mathrm{~mm}$ of water; we used ${ }^{24}$ a fixed total thickness of water of $24 \mathrm{~mm}$.

For the case of nondirectional reflected light, we defined $D_{\text {medNdir }}(\lambda)$, almost similar to the preceding case, but with 


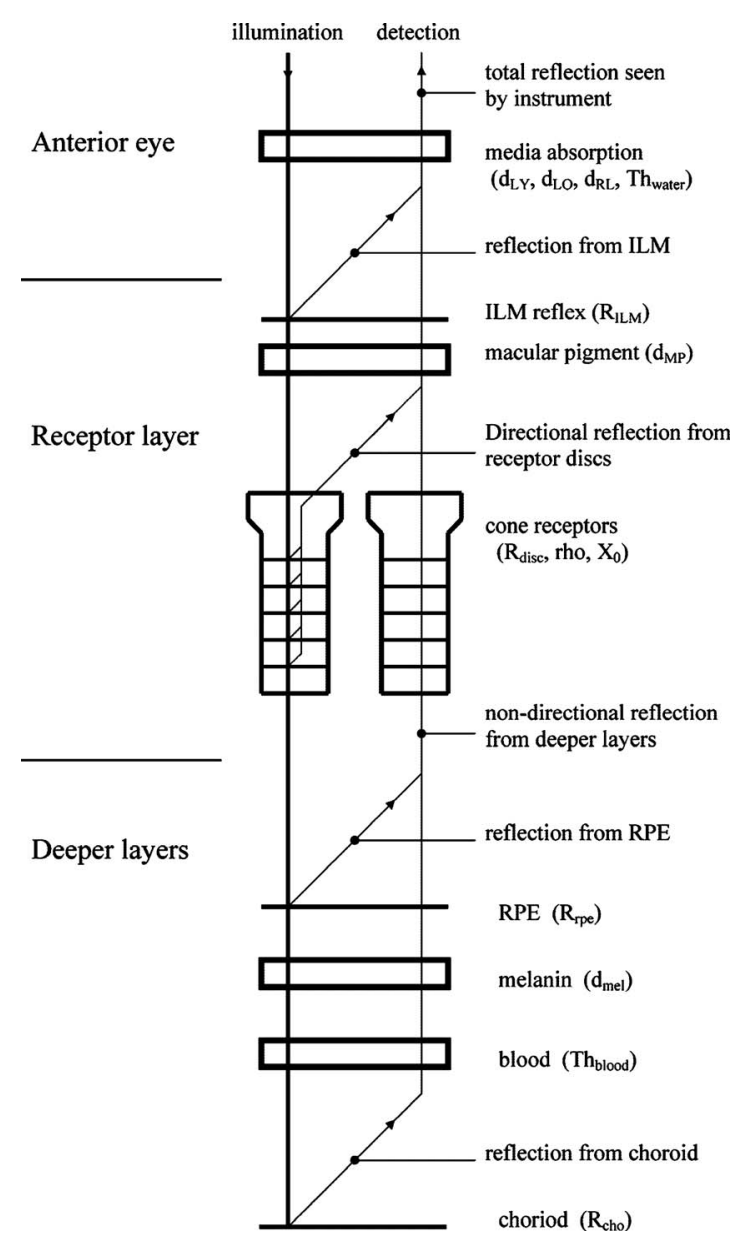

Fig. 4 Model of the reflection of the fovea. For clarity, separate pathways were drawn for light entering the eye and for reflected light. Light enters the eye at the left (thick line, illumination) and meets several layers of tissues in the anterior eye, the receptor layer, and the deeper layers posterior to the retina. Absorbing layers are shown as boxes, and reflecting layers are shown as horizontal lines. Reflection takes places at four layers and is symbolized by lines angled at $45 \mathrm{deg}$, continuing as the thin line at the right traveling upward to the detector. The only source of directional reflection seen by the instrument is from the cone discs, all others are nondirectional. Abbreviations are explained in the Secs. 3.2-3.5.

$d_{R L}$ set to zero. This assumption was made after fitting the data from the isolated nondirectional light in the pilot analysis, where $d_{R L}$ showed a strong tendency toward zero. The rationale is that the light that is scattered somewhere anterior to the cones changes angle of direction. It is therefore easily lost for the directional reflection, while it is not easily lost for the nondirectional component consisting of light scattered perhaps a second time in deeper layers. This assumption of regaining scattered light in the nondirectional condition was tested with nearly noiseless mean data from a young and old group (Sec. 5.3).

The transmission of the macular pigment was described by

$$
D_{M P}(\lambda)=d_{M P} \times M_{M P}(\lambda) .
$$

For the template of macular pigment absorption, we used a mixture of $70 \%$ zeaxanthin and $30 \%$ lutein $^{25}$ :

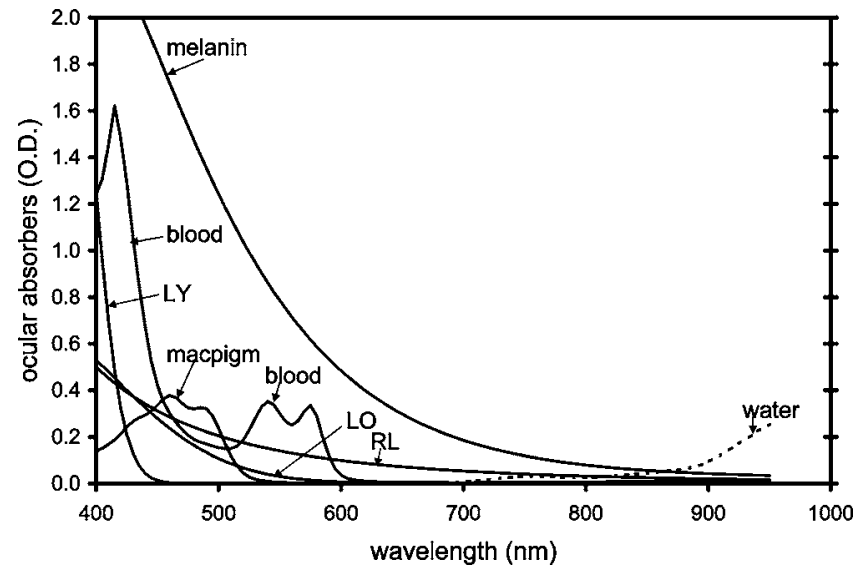

Fig. 5 Absorbers as appearing in the eye, displayed with the mean densities for our group of subjects. The absorbers in the eye media are LY (lens young), LO (lens old), RL (Rayleigh scatter losses), and above $800-\mathrm{nm}$ water. In the retina, macular pigment absorbs from 400 to $520 \mathrm{~nm}$. In the deeper layers, posterior to the retina, light is absorbed by melanin and blood.

$$
M_{M P}(\lambda)=0.7 * \operatorname{Zeax}(\lambda)+0.3 * \text { Lutein }(\lambda)
$$

We normalized it at the peak wavelength $(460 \mathrm{~nm})$ to 1 . Thus, parameter $d_{m p}$ is the density of macular pigment at $460 \mathrm{~nm}$.

\subsection{Directional Component}

The OSCE was described by a Gaussian shape in the pupil plane:

$$
\operatorname{SCE}(x)=10^{-\rho\left(x-x_{0}\right)^{2}},
$$

with $\rho$ the directionality, and $x$ the horizontal position relative to the entrance beam in the pupil plane of the instrument. Due to the alignment procedure (see Sec. 4.3), the position of the maximum of the OSCE at $x_{0}$ was always close to zero. Note first that we did not use a reference position relative to the center of the pupil as in the general form in Eq. (1). Note, too, that Eq. (1) also includes the nondirectional component.

$R_{\text {disc }}(\lambda)$, the directionally reflected light originating from the cone discs, was assumed to decrease with wavelength as

$$
R_{\text {disc }}(\lambda)=R_{\text {disc }} \times(550 / \lambda)^{2},
$$

with $R_{\text {disc }}$ the reflection from the outer segments. The wavelength dependency deviates from an earlier description ${ }^{5}$ for reasons addressed in Sec. 6.5.

At the level of the receptor layer, the directional reflection becomes

$$
R_{\text {recep }}(\lambda, x)=\operatorname{SCE}(x) \times R_{\text {disc }}(\lambda) .
$$

At the level of the cornea, the directional component in both the spectral plane and in the pupil plane can be described by

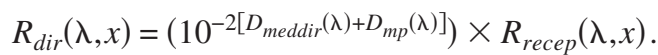

\subsection{Nondirectional Component}

The nondirectional component is easiest considered from the choroidal space. The backscattering of light in the choroidal 
van de Kraats and van Norren: Directional and nondirectional spectral reflection...

tissues was taken as a neutral reflection $R_{\text {choroid. }}$ Light in the choroidal space is absorbed by blood and melanin.

We defined the density of a layer of $95 \%$ oxygenated blood with a thickness of $100 \mu \mathrm{m}$ as $D_{\text {blood_100 } \mu \mathrm{m}}(\lambda){ }^{26}$ To account for the variety of path lengths through the center and periphery of small and large blood vessels, we assumed for the template a wedge-shaped blood layer, with path lengths from zero to $100 \mu \mathrm{m}$. These fixed path lengths were taken to avoid complex path length corrections during the fitting process. To obtain the final blood thickness parameter as a scalar in $\mu \mathrm{m}$, we divided the $100 \mu \mathrm{m}$ wedge results by 100 . The template for a layer of blood of $1 \mu \mathrm{m}$ with correction for different path lengths can now be calculated as

$$
\begin{aligned}
M_{\text {blood }}(\lambda)= & -0.01 \times \log \left\{\left[1-10^{-D_{\text {blood_100 } \mu \mathrm{m}}(\lambda)}\right] /\right. \\
& {\left.\left[D_{\text {blood_100 } \mu \mathrm{m}}(\lambda) \times \ln (10)\right]\right\} . }
\end{aligned}
$$

The density of a layer of blood with a thickness $T h_{\text {blood }}$ in $\mu \mathrm{m}$ is

$$
D_{\text {blood }}(\lambda)=T h_{\text {blood }} \times M_{\text {blood }}(\lambda) .
$$

For the transmission through a uniform layer of melanin, we took the spectral data of Gabel et al., ${ }^{27}$ approximated by

$$
\begin{aligned}
M_{m e l}(\lambda)= & 2.45 \times[0.007+0.165 \times \exp (-\lambda \times 0.0055) \\
& +113 \times \exp (-\lambda \times 0.011)-4300 \times \exp (-\lambda \\
& \times 0.022)] .
\end{aligned}
$$

The term 2.45 served to normalize the function to 1 at $500 \mathrm{~nm}$ so that $D_{m e l}$ is the parameter for the density of melanin at $500 \mathrm{~nm}$. The density of melanin becomes

$$
D_{m e l}(\lambda)=d_{m e l} \times M_{m e l}(\lambda) .
$$

Reflection from the deeper layers as seen at the level of the receptor layer is then described by

$$
R_{\text {deep }}(\lambda)=R_{\text {rpe }}+R_{\text {choroid }} \times 10^{-2 \times\left[D_{\text {blood }}(\lambda)+D_{\text {mel }}(\lambda)\right]},
$$

with $R_{r p e}$ the parameter for a neutral reflection at the RPE.

The complete description of the nondirectional reflection is

$$
\begin{aligned}
R_{\text {nondir }}(\lambda)= & 10^{-2 \times D_{\text {medNdir }}(\lambda)} \times\left[R_{I L M}+10^{-2 \times D_{m p}(\lambda)}\right. \\
& \left.\times R_{\text {deep }}(\lambda)\right],
\end{aligned}
$$

where $R_{i l m}$ represents a parameter for a neutral reflection at the ILM, and $D_{\text {medNdir }}(\lambda)$ is the special case for the density of the media without Rayleigh scatter losses, as explained in Sec. 3.2.

\subsection{Complete Model}

\begin{tabular}{|c|c|c|c|c|}
\hline $\begin{array}{l}\text { Parameter } \\
\text { (reference } \\
\text { wavelength) }\end{array}$ & $\begin{array}{c}\text { Value } \\
\text { (standard deviation) }\end{array}$ & $\begin{array}{l}\text { Correlation } \\
\text { with age }\end{array}$ & $\begin{array}{c}p \text {-value } \\
\text { for age } \\
\text { effect }\end{array}$ & $\begin{array}{l}\text { Repro } \\
(\%)^{\mathrm{b}}\end{array}$ \\
\hline$T h_{\text {water }}(\mathrm{mm})$ & 24 & - & fixed & - \\
\hline$d_{L Y}(400)$ & 1.260 & - & fixed & - \\
\hline$d_{L O}(400)$ & $0.530(0.305)$ & 0.890 & $<0.001^{a}$ & 3 \\
\hline$d_{R L}(400)$ & 0.500 & - & fixed & \\
\hline$R_{i l m}(\%)$ & $0.136(0.098)$ & -0.165 & 0.097 & 20 \\
\hline$d_{m p}(460)$ & $0.391(0.156)$ & 0.025 & 0.801 & 7 \\
\hline$R_{\text {disc }}(\%)$ & $2.121(0.917)$ & -0.657 & $<0.001^{a}$ & 8 \\
\hline$\rho\left(\mathrm{mm}^{-2}\right)(550)$ & $0.155(0.050)$ & -0.139 & 0.162 & 13 \\
\hline$d_{m e l}(500)$ & $1.245(0.222)$ & -0.049 & 0.625 & 1 \\
\hline$T h_{\text {blood }}(\mu \mathrm{m})$ & $65.36 \quad(31.69)$ & -0.516 & $<0.001^{a}$ & 6 \\
\hline$R_{r p e}(\%)$ & $0.563(0.155)$ & -0.037 & 0.712 & 9 \\
\hline$R_{\text {choroid }}(\%)$ & $7.918(1.441)$ & 0.221 & $0.025^{a}$ & 2 \\
\hline$X_{0}(\mathrm{~mm})$ & $-0.029(0.270)$ & - & - & - \\
\hline
\end{tabular}

At the level of the cornea, the total reflection is the addition of the directional component [Eq. (10)] and the preceding calculated nondirectional component [Eq. (16)].

$$
R_{\text {total }}(\lambda, x)=R_{\text {dir }}(\lambda, x)+R_{\text {nondir }}(\lambda) .
$$

The total number of parameters in the model is 13 (Table 1). In a single measurement, there are about 4500 free parameters (90 at 5-nm intervals in the spectral range from 400 to
Table 1 Mean parameters for the complete group of subjects.

$950 \mathrm{~nm} \times 50$ at $0.1-\mathrm{mm}$ intervals in the pupil range; see Sec. 4.1).

\section{Methods}

\subsection{Instrument}

The instrument described by Zagers et al. ${ }^{6}$ was redesigned in a desktop version that could be aligned with a joystick. In addition, the spectral range was extended to 400 to $950 \mathrm{~nm}$, and the switching mirrors were replaced by beamsplitters, enabling continued observation of both pupil and retina during measurements. A halogen lamp L (12-V, 30-W Wotan 64260, Osram, Munich, Germany) illuminated a spot of 1.8-deg diameter on the fovea (Fig. 6). The light was spectrally filtered by F ( 6 mm BG26 Schott AG, Mainz, Germany; 1-mm Schott UG3; Unaxis TL60, Linos, Goettingen, Germany) for the comfort of the subject and to prevent overloading the sensitive CCD camera (KX85 Apogee Instruments, Inc., Auburn, Massachusetts) that served as the detector. The intensity of the spot was $6.42 \log \mathrm{Td}$; calculations showed that it could be viewed safely for $15 \mathrm{~min}^{28}$ The filament of the halogen lamp was imaged in the pupil plane of the eye, where it defined the $2.6 \times 1.3 \mathrm{~mm}$ illumination pupil. With a separation of $0.7 \mathrm{~mm}$ below this illumination pupil, a slit-shaped detection pupil $\mathrm{S}$ of the instrument of $15 \times 1 \mathrm{~mm}$ formed the input for a prism-based imaging spectrometer. The $15-\mathrm{mm}$ slit length allows ample room for aligning an eye with a dilated pupil and an eccentric OSCE maximum (see Sec. 4.3). The 2-D 


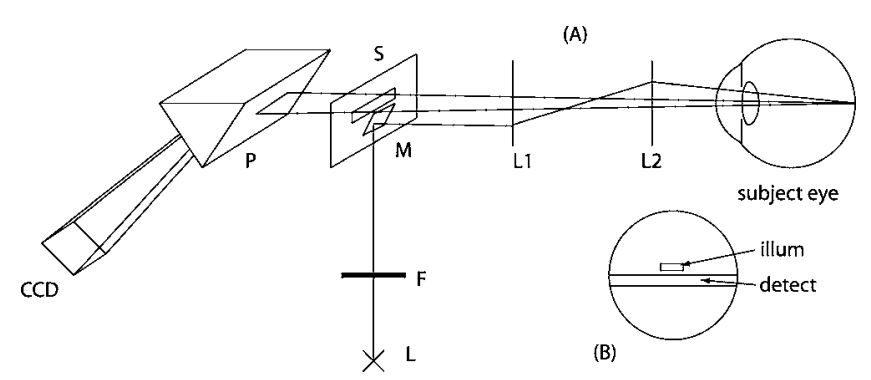

Fig. 6 (A) Simplified view of the instrument. $\mathrm{L}$ is a $30-\mathrm{W}$ halogen lamp. F are the filters to attenuate the red part of the spectrum. The lamp illuminates a spot at the fovea of $1.8 \mathrm{deg}$ via the small mirror $\mathrm{M}$, and the front lenses L1 and L2 which form a Badal system. L2 can be moved for focusing the retina. The reflected light from the retina (central $1.5 \mathrm{deg}$ ) fills the entire pupil, but only the part remaining after the slit $\mathrm{S}$ conjugated to the pupil is drawn. This light is spectrally decomposed by the dispersion prism $\mathrm{P}$ and forms a 2-D image at the CCD camera. (B) The area in the subject's pupil used for illumination of the fovea and the slit-shaped area used for detection of reflected light from the fovea.

image on the CCD detector had in one dimension the directional information from the intensity distribution over the slitshaped exit pupil. In the other dimension, it contained the spectral information, as light from each point of the slit was decomposed by the prism. Only light from the central 1.5-deg foveal spot was used for detection. Because of the prism, the image at the CCD with $1300 \times 1030$ pixels had an alinear spectral axis. It was transformed by software, using the dispersion calculation, to an intensity-normalized and linear reflection image of 250 pixels at $0.1-\mathrm{mm}$ intervals at the pupil axis, and 90 pixels at 5 -nm intervals at the spectral axis $(400$ to $950 \mathrm{~nm}$ ). Refraction errors were compensated by adjusting a Badal-type front lens system (L1 and L2).

\subsection{Calibration}

A mercury lamp (90-W, Type 93136, Philips, Eindhoven, The Netherlands) was used for spectral calibration. Before each measurement session, the instrument was calibrated for sensitivity by mounting two different calibration tubes on the front lens. An instrument stray-light image was assessed with a dark reference tube, containing a light trap. All subsequent images were corrected with this image. A white reference tube contained at the end a surface painted with white paint (Kodak 6080 White Reflectance Coating, Eastman Kodak Company, Rochester, New York). Because the distance of the white surface was at $230 \mathrm{~mm}$ from the instrument's pupil plane (10 times the focal distance of the eye), the measured white reference image represented a $1 \%$ reflection from the eye.

\subsection{Protocol}

A group of 102 subjects, mean age 49 years (range 18 to 75), took part in the measurements. The data of 53 subjects were published earlier using another analysis technique. ${ }^{29}$ The tenets of the Declaration of Helsinki were followed, and the local Medical Ethics Committee approved the protocol. Before the experiment started, the nature of the experiment was explained to the subjects, and written informed consent was obtained. The pupil of one eye was dilated with one or two drops of tropicamide $0.5 \%$. Subjects were aligned to the instrument, with the entrance beam clearly visible in the anterior eye. Initially, the entrance beam was placed somewhere above the center of the pupil, to allow space for the invisible detecting slit below the entrance beam. A chin rest and temple pads helped maintain the position of the head. The subjects were instructed to fixate the center of the illuminated spot. The front lens was adjusted for a sharp image of the retina at the monitor. Next, the subject leaned back, and the dark reference image was taken. Measurements at a rate of about 2 per second were displayed at the computer screen in the form of two cross sections of the 2-D image, the spectral shape at pupil profile position zero, and the pupil profile at $540 \mathrm{~nm}$. The alignment was optimized by searching in the pupil plane for the peak of the OSCE. This process was facilitated by simultaneously displaying (in a different color) the highest profile from the start of the measurements. The optimal horizontal position in the pupil plane was easily found by moving the instrument until the peak position of the Gaussian-shaped directional profile fell symmetrically around profile position zero. Profile position zero corresponds to the horizontal center of the entry beam. The optimal vertical position in the pupil plane was found by trial and error using the highest profile trace. During optimizing, the spectral view was watched to keep unwanted reflections from the anterior eye low. These reflections were recognized by their relatively high reflectances near $400 \mathrm{~nm}$. The optimization procedure took about 2 min, enough to bleach the visual pigments to almost $100 \%$. At the optimal position, five measurements with an integration time of $1 \mathrm{~s}$ each were obtained. If necessary, head position was readjusted when the head drifted away from the optimal position, recognized by a drop in amplitude.

\subsection{Pupil Limits}

The reflection profile, as seen in the entrance slit of the instrument, is cut off by the pupil of the eye. Left and right limits in these pupil plane positions were determined as follows. First, the data in the spectral range from 500 to $600 \mathrm{~nm}$ (where the signal has low noise) were binned to $5 \mathrm{~nm}$, yielding 20 profiles. Next, left and right from the central pupil position, those data points where the signal dropped to $20 \%$ of the central value were taken. From those 20 left and right limits, we took the lowest. In the final step, the left and right limits were moved $1 \mathrm{~mm}$ to the center and applied to the complete measurement to avoid any interference from the pupil edge.

\subsection{Subselection of Young and Old Groups}

To develop and evaluate the model, two subselections were made from the group of subjects: a group $<40$ years (mean 24 ), and a group $>50$ years (mean 58). From the five measurements per subject (see Sec. 4.3), a further selection was made by allowing measurements only over at least $4 \mathrm{~mm}$, with pupil limits outside the range $-2.5,+1.5 \mathrm{~mm}$ (nasal to temporal), and a minimum of three measurements per subject. This gave 45 measurements from 10 subjects for the young group and 81 measurements from 18 subjects for the old group. To find a template for $\rho$ as a function of wavelength, the pupil profiles at every wavelength (binned to $5 \mathrm{~nm}$ ) for the young group were analyzed for $\rho$ with the Gaussian model from Eq. (1). The means of the standard deviations within a 

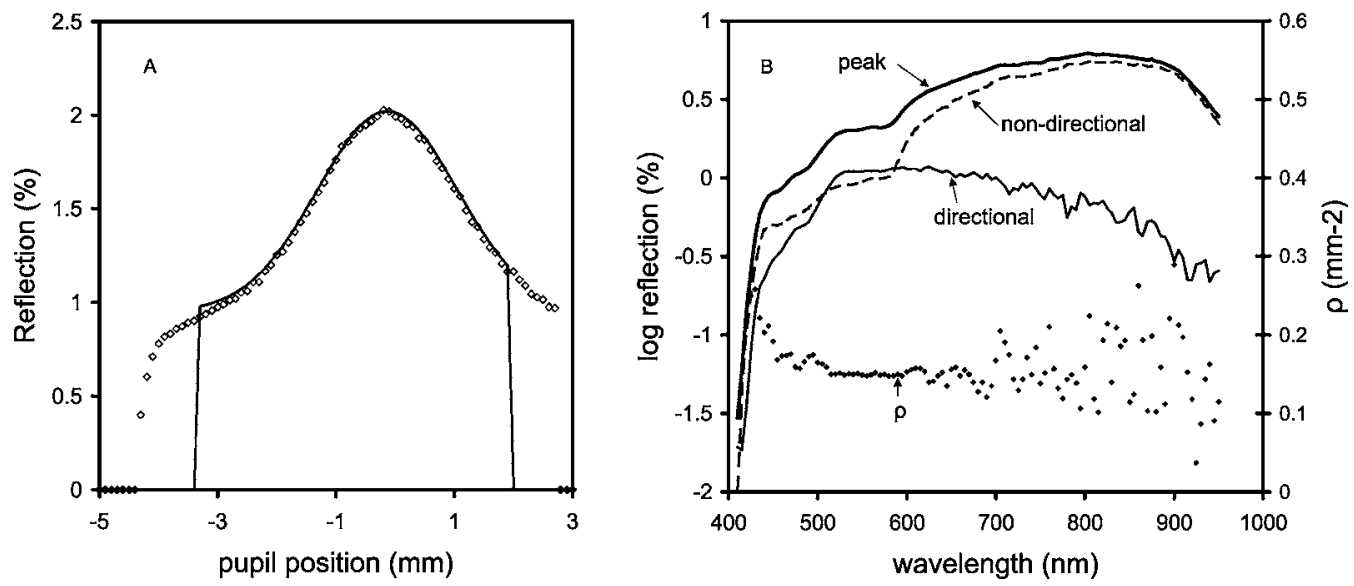

Fig. 7 Analysis of a single measurement using the Gaussian fit from Eq. (1). (A) Data points of the pupil profile at $540 \mathrm{~nm}$ (error bars smaller than the symbols) and model curve (solid line). (B) Spectral behavior of the directional reflection from the foveal cones (thin solid curve), the nondirectional reflection (dashed curve) from the background, originating from pre- and post-receptor layers, and the sum of both (thick solid line). Continuous curves connecting the data points are presented for clarity. The data points in the lower half of the figure represent $\rho$ (scale on the right). Because of the noisy appearance and to avoid overlap with the amplitude curves, $\rho$ is not plotted below $420 \mathrm{~nm}$. In such a single measurement, the decline of $\rho$ with wavelength is not clear and can deviate from the mean result presented in Fig. 2 .

subject were calculated to provide an indication for the error in the data points. Next, a "young measurement" was calculated by averaging the data points in the young group within the pupil range they had in common in their original measurements; similarly, an "old measurement" was calculated.

\subsection{Fitting the Data}

For the pilot explorations with the young and old group data, the Solver in Microsoft Excel 2003 was used to fit the data with the model by minimizing chi-squares. All spectral templates were convolved with the bandwidth of the spectrometer. For the final analysis of individual measurements with the 2-D model, the Levenberg-Marquardt routine ${ }^{30}$ was applied. The noise in the data points, used for weighting, was calculated from the square root of the photons counted. For analyzing the statistics, SPSS 12.0.1 for Windows (SPSS, Inc., Chicago) was used.

\section{Results}

\subsection{Single Subject}

To illustrate the spatial profile and the spectral behavior of the directional and the nondirectional components, the data from a single measurement of a 20 -year-old subject are presented in Fig. 7. The amplitudes of the directional component and the nondirectional component were estimated by fitting the elevated Gaussian curve from Eq. (1), at each wavelength to the pupil profile. The result at $540 \mathrm{~nm}$ is presented in Fig. 7(A). The amplitudes versus wavelength are presented in Fig. 7(B). The total reflection at the peak of the profile was found by adding the amplitudes of the directional component and the nondirectional component. Going from left to right, both curves show a sharp increase beyond $420 \mathrm{~nm}$, where the lens absorption ends (young eye), and a further increase near $500 \mathrm{~nm}$, where the absorption from the macular pigment (MP) ends. The effect of macular pigment is stronger in the directional curve than in the nondirectional one. The nondirectional curve displays an increase near $600 \mathrm{~nm}$, where blood no longer absorbs, together with a gradual increase due to increasingly less absorption in melanin. The directional curve lacks these features. Beyond $700 \mathrm{~nm}$, the curves show the influence of absorption by water, leading to a strong decline at $900 \mathrm{~nm}$.

The more complex shape of the nondirectional component due to the addition of light from the choroid, visible above $580 \mathrm{~nm}$, is evident. The dilution of the macular pigment fingerprint near $500 \mathrm{~nm}$, due to preretinal reflectors, is also clearly visible.

When the directional component becomes low in the absolute sense, or low relative to the nondirectional component, the estimation of $\rho$ becomes increasingly noisy, visible below 500 and above $600 \mathrm{~nm}$. Through interaction in parameters, noise in $\rho$ then also causes noise in the separation of the directional and nondirectional amplitude. To resolve this, a smooth template for $\rho$ as function of wavelength was derived (Sec. 5.2). The single parameter for $\rho$, instead of a value at each wavelength, also keeps the total number of parameters in the model limited to 13 instead of more than 100 .

\section{$5.2 \rho$ Template}

The mean $\rho$ in the young group as a function of wavelength (Fig. 2) was used to find the parameters $\rho_{w g}$ and $\rho_{\text {scatt }}$ in Eq. (2), resulting in

$$
\rho(\lambda)=0.050+0.097 \times(550 / \lambda)^{2} .
$$

A similar analysis of the old group yielded similar results in the spectral range from 500 to $600 \mathrm{~nm}$. Below $500 \mathrm{~nm}$, the result was very noisy due to the high density of the aged lens. Also, at the longer wavelengths above $600 \mathrm{~nm}$ with the increasing amplitude of the nondirectional component, the more irregular shape of the reflection in the pupil plane in the older group appeared as noise in the directional component. The error bars in Fig. 2 are the means of the standard deviations within a subject. These values were used for weighing the data in the fitting process. Equation (18) normalized to 1 at 

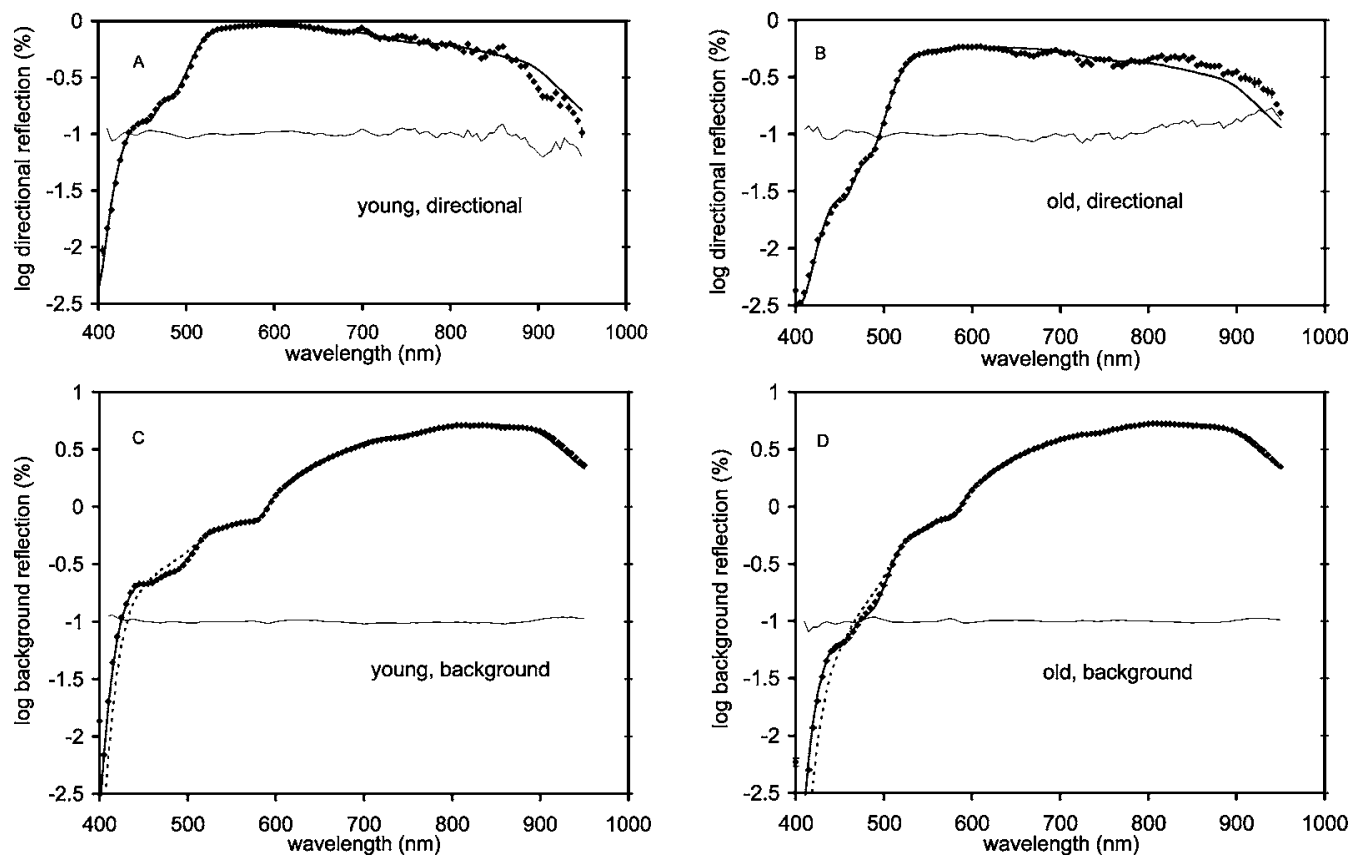

Fig. 8 Mean (log) reflection as a function of wavelength for the young (left) and old (right) groups. The upper panels show the directional reflection from the cones, and the lower panels show the nondirectional background reflection from layers anterior and posterior to the receptor layer. The data points have error bars with the mean standard deviation, but most are smaller than the symbols. The model curve (thick line) almost exactly replicates the data points. The residue, the difference between the data points and the model, is shown as a thin line; it was shifted downward 1 log unit for easy viewing. In the lower panels, the fit with the Rayleigh losses $d_{R L}$ set to the same value as found in the directional fitting, instead of set to zero, is shown for comparison (dotted line, no residue shown).

$550 \mathrm{~nm}$ was used as a template for $\rho$ as a function of wavelength.

\subsection{Test of $d_{R L}$ on Mean Directional and Nondirectional Spectra for the Young and Old Groups}

The almost noiseless mean data from the young group and the mean data from the old group were used to test the general concept of the spectral model from Sec. 3, and in particular the assumption of $d_{R L}=0$ for the nondirectional part. With the template for the spectrum of $\rho$ [Eq. (18)], data were fit using Eq. (1) to derive the directional and nondirectional amplitudes. Results of these analyses are shown as the data points in Fig. 8. Model curves were drawn using the equations from Secs. 3.3 and 3.4. The directional data were fitted first to Eq. (10) (with $d_{R L}$ and $d_{L Y}$ fixed to the calculated values according to the mean age of the groups), yielding values for $d_{L O}$, $d_{M P}$, and $R_{d i s c}$. The nondirectional data were fitted next to Eq. (16) (with $d_{L O}, d_{M P}$, and $R_{\text {disc }}$ fixed to the values obtained in the directional fit, and $d_{L Y}$ fixed as above and $d_{R L}$ set to zero), yielding values for $d_{\text {mel }}, T h_{\text {blood }}$, and $R_{\text {ilm }}, R_{\text {rpe }}$, and $R_{\text {choroid }}$.

An almost perfect fit to the data could be obtained (chisquare young $=17$; chi-square old $=17$ ). When $d_{R L}$ was not set to zero for the fitting of the nondirectional spectra, but also taken as a fixed parameter according to the mean age of the groups [by replacing $D_{\text {medNdir }}$ in Eq. (16) by $D_{\text {meddir }}$ ], chisquare values were more than an order of magnitude higher (chi-square young=244; chi-square old=257). The two-step strategy of first fitting the media and macular pigment parameters on the directional component, and so avoiding interac- tion with deeper layer parameters, was applied in this section only to strengthen the assumption of setting $d_{R L}$ to zero for the nondirectional component. In the rest of this paper, fitting to the data of individual measurements, all free parameters were fitted simultaneously.

\subsection{Age Effects}

Next, the model was applied to the individual measurements, allowing the study of age effects in the parameters. In total, 510 measurements from 102 subjects were analyzed. At first, all 13 parameters were allowed to vary, except water (fixed to $24 \mathrm{~mm}$ ). As expected, the media term $d_{L O}$ showed a high correlation with age $(0.843, p<0.001)$. The media parameter $d_{L Y}$, however, showed an unexpected negative trend with age. ${ }^{23}$ Inspection of the original data showed that with the increased density of the lens at higher ages $\left(d_{L O}\right)$ together with the attenuation by $d_{R L}$, the measured reflection near $400 \mathrm{~nm}$ reached the noise floor of the instrument. This limited the necessary dynamic range for proper detection of $d_{L Y}$. Other parameters have a much wider effective spectral range of their templates and were less sensitive to this problem. In the receptor layer, only $R_{\text {disc }}$ showed a significant correlation with age $(-0.466, p<0.001)$. In the deeper layers, $T h_{\text {blood }}$ reached the value $-0.487(p<0.001)$. Last, a very low correlation was found for $R_{\text {choroid }}(0.209, p=0.035)$.

The media scatter term $d_{R L}$ showed a low (but not significant, $p=0.551)$ correlation with age, as found in an earlier study. ${ }^{23}$ To avoid problems due to the low signal at $400 \mathrm{~nm}$, both media parameters $d_{L Y}$ and $d_{R L}$ were set to the calculated values for a mean age of $40,{ }^{23}$ leaving $d_{L O}$ as a free parameter 

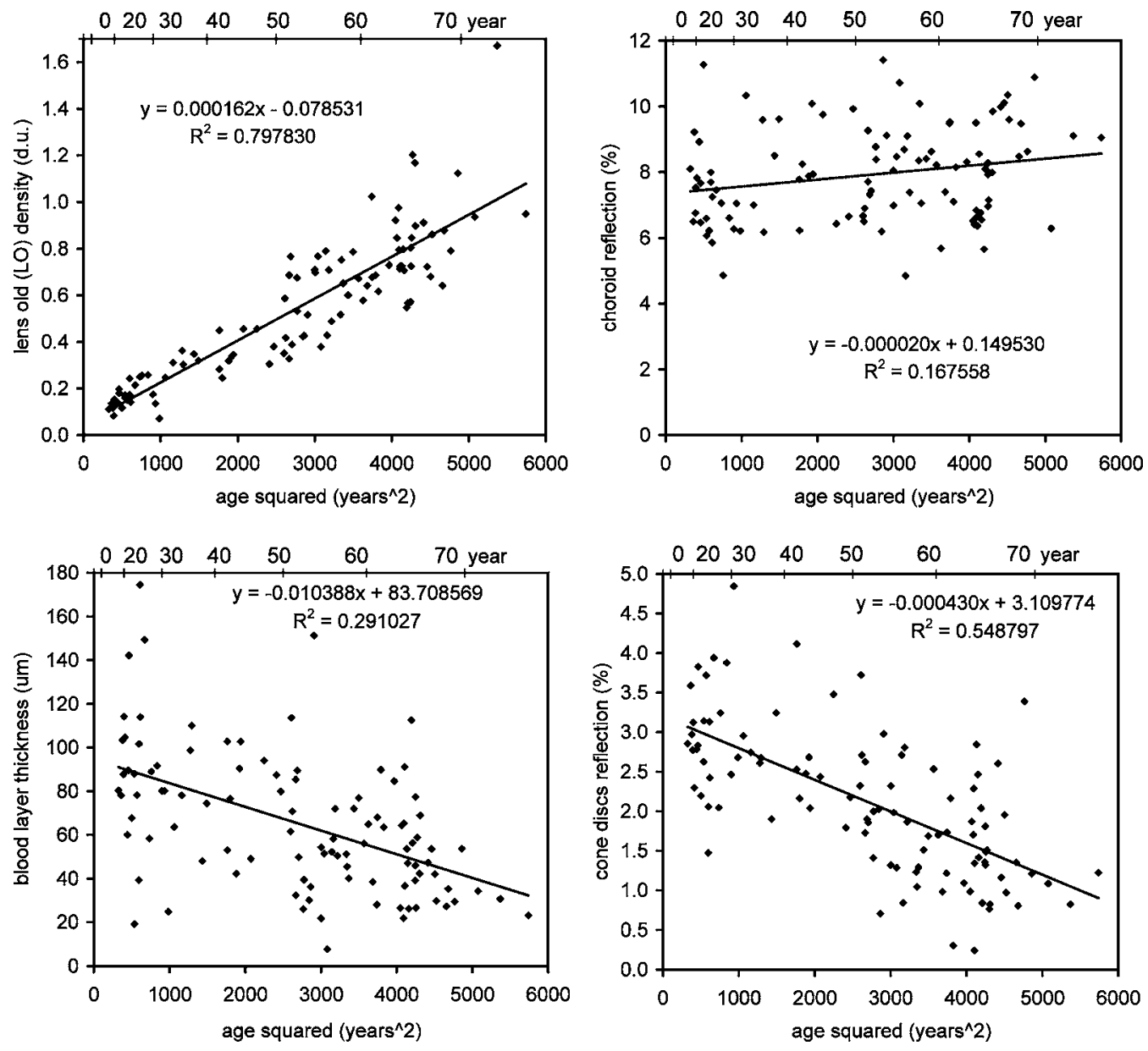

Fig. 9 Age trends for the parameters lens old, choroid reflection, blood layer thickness, and cone discs reflection.

for the media. This left 10 free parameters for the final fitting of the data. The mean results are summarized in Table 1. Parameters with a significant age trend $(p<0.05)$ were plotted versus age squared in Fig. 9. Age squared was used because it consistently yielded smaller chi-squares than a linear fit. $^{23}$ Reproducibility (the repro column in Table 1) was defined as the average coefficient of variation $(100 \times$ standard deviation in the five measurements per subject/mean parameter value).

\subsection{Parameter Cross Influences}

The mean covariances between parameters of all individual measurements were calculated from covariances provided by the fit routine. Only three appeared higher than 0.6: the value 0.694 between $\rho$ and the reflection from the RPE, 0.681 between the ILM reflection and the density of macular pigment, and 0.620 between melanin and the reflection from the choroid.

The Pearson bivariate correlations between parameters after correction for age were also calculated. Neglecting correlations below 0.266 , corresponding to $7.1 \%$ of the variance explained, only two remained with $p<0.05$. The first is between $\rho$ and $R_{\text {rpe }}(0.545)$, the second between melanin and blood $(-0.663)$.

\section{Discussion}

\subsection{General}

We developed a model for fundus reflection that for the first time simultaneously describes the spectra of the directional and nondirectional reflection of the human fovea at a high spectral and spatial resolution. The excellent fits support the assumption that the deeper layers generate no directional component. Van de Kraats et al. ${ }^{5}$ already gave experimental and theoretical evidence, and this was also confirmed by Burns et al. ${ }^{14}$ using the autofluorescence of lipofuscin. As the fluorescence is by nature omnidirectional, it formed a new source of light behind the receptor layer, undisturbed by preretinal reflections. We found that a large part of the light from deeper layers must be recaptured by the cones due to the reciprocity principle (Sec. 2.4). At first glance, a surprising aspect of the model is that Rayleigh scatter losses in the eye media and in preretinal layers have to be included only in the directional pathway. This will be discussed in the next section.

\subsection{Absorbers}

\subsection{Media}

In the initial analysis of the complete group of 102 subjects, the lens young component $d_{L Y}$ and the Rayleigh scatter com- 
van de Kraats and van Norren: Directional and nondirectional spectral reflection...

ponent $d_{R L}$ were free parameters. The mean value of $d_{L Y}$ was $0.61 \pm 0.35$, and $d_{R L}$ was $0.55 \pm 0.33$. In a pilot analysis, the reflection near $400 \mathrm{~nm}$ appeared so low due to the combined attenuation caused by $d_{L Y}$ and $d_{R L}$ that it reaches the noise level of the instrument, limiting the dynamic range for proper detection. In a second analysis, therefore, $d_{L Y}$ and $d_{R L}$ were fixed to mean values corresponding to an age of 40 years (1.26 and 0.5$).^{23}$ This prevented less meaningful results, especially at older ages when $d_{L O}$ increases substantially. The decrease with age of the lens young component as shown by Zagers probably was contaminated by this effect. ${ }^{21}$ Going from 20 to 60 years, the density for the total media at $420 \mathrm{~nm}$ in the Zagers study increased by $0.12 \mathrm{log}$ units. Our data showed an increase of $0.46 \log$ units at $420 \mathrm{~nm}$. This suggests that the Zagers increase in age of the lens old component was compensated by a too large decrease of the lens young component.

The significant improvement of the fits by using the value for $d_{R L}$ of 0.5 in the directional reflection, but setting it to zero in the nondirectional one, was surprising at first. At second thought, it was explained by part of the light that is scattered anterior but near to the cones. Light scattered in that layer is changing the angle of direction, and therefore is easily lost for the directional reflection, while it is not easily lost in the case of the diffusely scattered nondirectional component. Van den Berg et al. ${ }^{31}$ presented optical scatter losses in the cornea, but in fact they included the humors and preretinal layers as well. Their magnitude of the Rayleigh scatter losses for a 1-deg field can be calculated as 0.34 . Estimates of losses of light scattered outside a retinal field of 1 -deg by the cornea and lens for the relatively young eye from Vos et al. ${ }^{32}$ result in a density of only 0.03 . For our larger illumination field of $1.8 \mathrm{deg}$, it should be even less. According to this, a large part of our Rayleigh scatter value of 0.5 originates from additional scatter losses in preretinal layers anterior to the cone photoreceptors. The model is however unable to discriminate between these losses and scatter losses in the eye media.

The aging slope of $0.000162 \times$ age $^{2}$ for the media attributed here solely to $d_{L O}\left(d_{R L}\right.$ was fixed) is comparable to the sum of the aging values $d_{L O}$ and $d_{R L}(0.000132+0000031$ $=0.000163$ ) from a literature study. ${ }^{23}$ Apparently because of fixing the value of $d_{R L}$ to 0.5 , some of its (small) aging aspect has moved into $d_{L O}$.

\subsubsection{Macular pigment}

The macular pigment optical density in a subgroup of 53 subjects was analyzed before with two previous models and compared with densities obtained with heterochromatic flickerphotometry (HFP), scanning laser ophthalmoscope (SLO), reflectance difference at 488 and $514 \mathrm{~nm}$, and SLO autofluorescence. ${ }^{29}$ Analysis of the data with the 1996 version of the fundus reflection model $^{5}$ resulted in 0.44 , compared with the current 0.37 . A simple model containing only the directional component ${ }^{21}$ yielded 0.53 , being too high because of the earlier mentioned parameter interaction with the Zagers lens templates. ${ }^{21}$

Apart from the current wavelength-dependent reflection from the cones, some of the differences can be due to the different absorption data for macular pigment used. The Handelman data ${ }^{25}$ we used show a somewhat different density
Table 2 Macular pigment density using different methods.

\begin{tabular}{lccc}
\hline Method & $\begin{array}{c}\text { Field size } \\
(\mathrm{deg})\end{array}$ & $d_{\text {MP }}$ & $\begin{array}{c}\text { Correlation } \\
\text { with current }\end{array}$ \\
\hline Current & 1.5 & 0.37 & 1 \\
Kraats 1996 & 1.5 & 0.44 & 0.95 \\
Zagers 2004 & 1.5 & 0.53 & 0.88 \\
SLO reflectance & 0.5 & 0.33 & 0.66 \\
SLO auto-fluoresence & 0.5 & 0.31 & 0.61 \\
HFP & 1 & 0.30 & 0.57 \\
\hline
\end{tabular}

near $420 \mathrm{~nm}$ (see Sec. 3.2) than previously used data. Comparing the absolute numbers with results from other instruments (Table 2) is also more difficult because of the strong effect of the retinal area under test (here, $1.5 \mathrm{deg}$ ). Unexpected at first sight, the models, while using a larger field, yield the highest densities. This is explained by the fact that the models compensate for all disturbing factors, like the diluting effect from light reflected at the ILM. The necessity for a good model is seen in Fig. 7(B), where the macular pigment fingerprint is unmistakably higher in the directional component compared to that of the nondirectional component. The latter clearly shows the diluting influence of the ILM reflection. The SLO reflection method was lacking such compensation. More difficult to understand is the lower value for the SLO autofluorescence technique, because here dilution by preretinal autofluorescence is not expected.

We repeated the calculation of the Pearson bivariate correlations on macular pigment between the current model results, previous models, and the other techniques (Table 2). All correlations were highly significant $(p<0.001)$. The $d_{M P}$ parameter showed no significant change with age, as in most other studies, ${ }^{29,33,34}$ with the notable exception of a recent study including over 800 subjects reported by Nolan et al. using HFP that showed an unexpected decrease of 0.17 from 20 to 60 years. $^{35}$

For the complete group of 102 subjects, despite the covariance of macular pigment with the reflection from the ILM of 0.681 , the model seemed to succeed well in separating them, as shown by the low and not significant correlation after correction for age $(0.113, p=0.259)$.

\subsubsection{Blood}

For the absorption in blood, we used a template derived from a $100-\mu \mathrm{m}$ wedge-shaped layer of blood. This is a more realistic model of the blood layer, because it contains a large range of path lengths through the blood layer instead of always the same length as with a homogeneous layer thickness. The result is that the large dynamic range in the transmissions over the whole spectrum in the case of a homogeneous layer thickness is compressed, yet without adding more parameters to the model. ${ }^{3,36} T h_{\text {blood }}$ declines significantly with age, probably due to the accumulation of basal laminar deposits on the 
choriocapillaries and Bruchs membrane, ${ }^{37-39}$ screening the choriocapillaris.

Measurements of choroidal blood volume with a laser Doppler flow technique also shows a decrease of about $45 \%$ from 20 to 70 years. $^{40}$ Another study using morphometric analysis showed a $45 \%$ decrease in the density and a $34 \%$ decrease in the diameter of the lumen of the choriocapillaris from the first to the tenth decade. ${ }^{41}$ It is difficult to say how these phenomena impact on $T h_{\text {blood }}$, and what fraction of $T h_{\text {blood }}$ is from the choriocapillaris.

The correlation between blood and melanin of -0.663 is likely to have a true physiological background. Their templates are so different that the interaction of parameters in the model is not expected. This is expressed by the low covariance between these parameters (0.138). One obvious explanation is that blood and melanin in some degree crowd each other out.

\subsubsection{Melanin}

Melanin was not found to be age dependent. This is a minor contradiction with determinations from young and old donor material based on only the retinal pigment epithelium, decreasing about $37 \%$ in the macular area. ${ }^{42}$ In analyzing the results of the macular area of only 16 subjects in another study, an age relation could not be found. ${ }^{43}$ Measured in optical density units, the fraction of the melanin in the RPE is about one-third of the total melanin. ${ }^{27,44}$ If two-thirds of the melanin fraction in the choroidal space is constant with age, age dependency is expected to be much lower. While pure melanin decreases with age, more complex granules of the type melanolipofuscin and melanolysosomes increase by $72 \% .{ }^{42}$ With their absorption spectrum not very different from pure melanin, ${ }^{45}$ discrimination with our reflection technique is not possible-another argument for an almost zero age dependency of melanin. More important is that in the case of using reflection techniques to derive the total melanin content, it better reflects the functionality of melanin for the stray-light reduction in the retina. Other techniques based on pure melanin only are perhaps less important in this respect.

Despite the covariance of melanin with the reflection from the choroid of 0.62 , the model seemed to succeed well in separating them, as shown by the low correlation after correction for age $(0.252, p=0.011)$.

\subsection{Nondirectional Reflectors}

The first, small reflection (approximately $0.1 \%$ ) occurs at the ILM. A reflection at the outer limiting membrane was not incorporated because it cannot be discriminated from the reflection $R_{\text {rpe }}(0.56 \%)$ located at the receptor layer/retinal pigment layer interface. Although a reflection at Bruchs membrane seems physiologically probable, it would add at least two parameters $\left(R_{\text {bruch }}\right.$ and $\left.D_{\text {RPEmelanin }}\right)$ to the deeper layers, as in Delori et al. ${ }^{3}$ Without these extra parameters, we obtained very good fits. The price to be paid is that the absolute values of $d_{m e l}$ and $T h_{\text {blood }}$ have limited physiological meaning; trends are relevant, however. Reflection of the choroid increases somewhat with age (Fig. 9), possibly due to the same deposits as discussed for the increases of blood layer thickness with age.

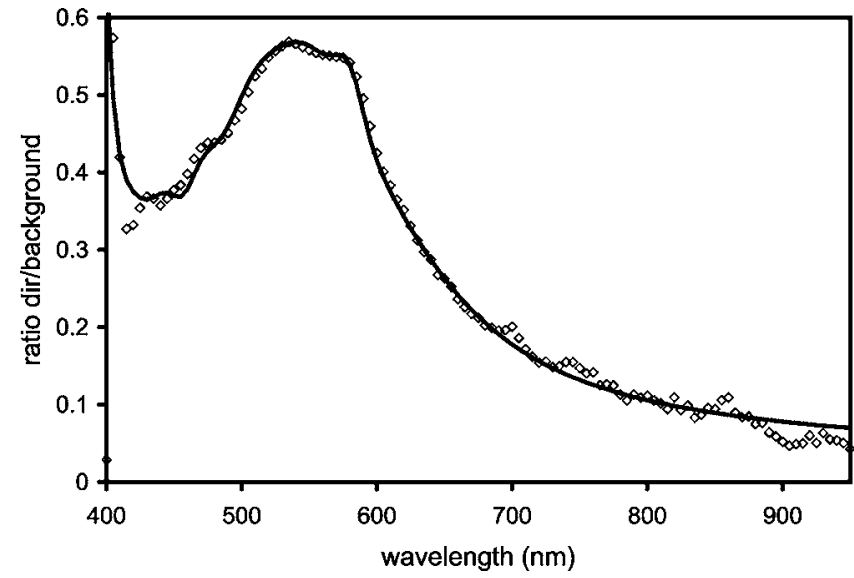

Fig. 10 The directional fraction of the total light reflected from the fovea versus wavelength for the young group. Maximum directionality is around $525 \mathrm{~nm}$. At lower wavelengths, the directional cone signal is reduced by macular pigment, while the ILM part of the nondirectional reflection is not reduced. At longer wavelengths, there is a growing influence from the nondirectional reflection from the deeper layers because blood and melanin become more and more transparent.

\subsection{Directional Reflection}

In previous papers, we assumed that the origin of the cone directional reflection lay in spectrally neutral Fresnel reflections by small differences in the refractive index of the discs and the interstitional fluids. ${ }^{5,21,46}$ Although this was supported by measurements of Zagers et al. on pseudophakic subjects, ${ }^{21}$ Choi et al. ${ }^{47}$ argued that the wavelength range used was too limited to support the conclusion. Other authors ${ }^{48,49}$ assumed the integral volume of the Gaussian shape to show spectrally neutral behavior, as is commonly found in diffraction-limited processes. ${ }^{16}$ Consequently, the peak amplitude should drop with lambda ${ }^{-2}$. With the current, much-extended wavelength range, the peak amplitude was clearly seen to decrease at the longer wavelengths, where other absorbers play no role [Figs. 7 (B) and 8]. We therefore also opted for lambda ${ }^{-2}$ behavior in the new model. Following Marcos et al. ${ }^{9,10}$ the $\rho$ of the waveguiding part of the OSCE is constant with wavelength. Another scatter component from interference of light from neighboring cones introduces lambda ${ }^{-2}$ behavior, as seen in Fig. 2. Summarizing, we have shown that the light intake by the cones on perpendicular entrance is neutral because of the large capture area (Sec. 2.4). The summated reflection from the discs itself still remains a neutral Fresnel reflection. The light escaping the receptor in the backward direction forms a Gaussian intensity distribution at the pupil plane, with the properties shown in Figs. 1 and 2. The volume, however, is taken as neutral.

Choi et al. ${ }^{47}$ stated, on the basis of three wavelengths (550, 650 , and $750 \mathrm{~nm})$, that the guided fraction under the 2-D OSCE is neutral with wavelength. Our extensive wavelength data (Fig. 10) follow the model curve and clearly show a strong wavelength dependency (note that absorption in the media is cancelled in the calculation); a maximum is at around $525 \mathrm{~nm}$. Such wavelength dependence is obvious because below $525 \mathrm{~nm}$, the directional cone signal is reduced by macular pigment, while the ILM part of the nondirectional 
reflection is not. At wavelengths above $580 \mathrm{~nm}$, the nondirectional reflection from the deeper layers increases because blood and melanin become more and more transparent.

The correlation between $\rho$ and $R_{\text {rpe }}$ of $0.545(p<0.001)$ could in principle be caused by a non-Gaussian behavior in the skirts of the directional reflection. If the theoretical function is low in that region compared to the real directional profile, it uses $R_{\text {rpe }}$ to fill the gap. Physiological interaction is less likely with the relatively high covariance of 0.694 in mind, an indication that the model likes to interact with these parameters. A practical solution in further applying the model could be fixing $\rho$ and using $R_{\text {disc }}$ as the indicator of healthy cones.

For the present purpose with bleached visual pigments, $R_{\text {disc }}$ might as well be taken as the outer-segment end reflection. That the discs are the origin could only be made plausible by experiments with dark adapting and bleaching of the visual pigments. ${ }^{5}$ Figure 9 shows a large decrease in the disc reflection as a function of age. That the large reflection from the choroid $(8 \%)$ and that from the retinal pigment epithelium $(0.56 \%)$ have no such a decrease is a strong argument for the receptors as origin. Because the width of the Gaussian as given by $\rho$ has no age dependency and there is neither evidence for a large decrease in the number of cones nor for a large decrease in length of the outer segments, ${ }^{17,50}$ we might speculate that the indexes of refraction of the discs, and the interspaces between them, slowly drift toward each other at older ages. In diseases like age-related macular degeneration (AMD), $R_{\text {disc }}$ might decrease at a faster rate because here the number of cones or the lengths of the outer segments are possibly affected.

\section{Conclusion}

A new model gives fundamental insight in to how the directional and nondirectional spectral reflection from the fovea are related to each other. Previous attempts were less successful because the transmission in the eye media was taken as equal in both components. Setting the Rayleigh scatter to zero in the nondirectional reflection component caused a dramatic improvement of the model fit. This part is, however, presently not fully understood; we could not find support from other sources in the literature for additional scatter losses in preretinal layers. Further work on this issue will be needed.

The model generates quantitative estimates of several important optical absorbers in the eye as macular pigment, lens density, blood, and melanin, as well as parameters related to the optical quality of the cones, the cone disc reflection, and $\rho$. In addition, the model estimates the reflection from the retinal pigment epithelium and the choroid. A validation of the macular pigment optical density was obtained by the significant correlations obtained with independent estimates.

\section{Acknowledgments}

The authors are indebted to Dr. Tos Berendschot, Martijn Kanis, and Robert Wisse for allowing us to use their data.

\section{References}

1. D. van Norren and L. F. Tiemeijer, "Spectral reflectance of the human eye," Vision Res. 26, 313-320 (1986).
2. G. J. van Blokland and D. van Norren, "Intensity and polarization of light scattered at small angles from the human fovea," Vision Res. 26, 485-494 (1986).

3. F. C. Delori and K. P. Pflibsen, "Spectral reflectance of the humanocular fundus," Appl. Opt. 28, 1061-1077 (1989).

4. D. van Norren and J. van de Kraats, "Retinal densitometer with the size of a fundus camera," Vision Res. 29, 369-374 (1989).

5. J. van de Kraats, T. J. M. Berendschot, and D. van Norren, "The pathways of light measured in fundus reflectometry," Vision Res. 36, 2229-2247 (1996)

6. N. P. A. Zagers, J. van de Kraats, T. T. J. M. Berendschot, and D. van Norren, "Simultaneous measurement of foveal spectral reflectance and cone-photoreceptor directionality," Appl. Opt. 41, 4686-4696 (2002).

7. J. Krauskopf, "Some, experiments with a photoelectric ophthalmoscope," in Excerpta Medica, International Congress Series, M. A. Bouman and J. J. Vos, Eds., pp. 171-181, Excerpta Medica, Amsterdam (1965).

8. R. A. Applegate and V. Lakshminarayanan, "Parametric representation of Stiles-Crawford functions: normal variation of peak location and directionality," J. Opt. Soc. Am. A 10, 1611-1623 (1993).

9. S. Marcos, S. A. Burns, and J. C. He, "Model for cone directionality reflectometric measurements based on scattering," J. Opt. Soc. Am. A 15, 2012-2022 (1998)

10. S. Marcos and S. A.Burns, "Cone spacing and waveguide properties from cone directionality measurements," J. Opt. Soc. Am. A 16, 9951004 (1999).

11. N. P. A. Zagers, T. T. J. M. Berendschot, and D. van Norren, "Wavelength dependence of reflectometric cone photoreceptor directionality," J. Opt. Soc. Am. A 20, 18-23 (2003).

12. S. A. Burns, J. C. He, and F. C. Delori, "Do the cones see light scattered from the deep retinal layers," Vision Science and Its Applications, Vol. 1 of 1997 OSA Technical Digest Series, Optical Society of America, Washington, DC, pp. 94-97 (1997).

13. P. M. Prieto, J. S. McLellan, and S. A. Burns, "Investigating the light absorption in a single pass through the photoreceptor layer by means of the lipofuscin fluorescence," Vision Res. 45, 1957-1965 (2005).

14. S. A. Burns, F. C. Delori, and J. C. He, "Back-illuminating the cones: is the light from the RPE guided?" Invest. Ophthalmol. Visual Sci. 38 ARVO abstract No. 57 (1997).

15. F. C. Delori, D. G. Goger, B. R. Hammond, D. M. Snodderly, and S. A. Burns, "Foveal lipofuscin and macular pigment," Invest. Ophthalmol. Visual Sci. ARVO abstract No. 38, 1657, 1 (1997).

16. A. E. Siegman, "The antenna properties of optical heterodyne receivers," Appl. Opt. 5, 1588-1594 (1966).

17. C. A. Curcio, K. R. Sloan, R. E. Kalina, and A. E. Hendrickson, "Human photoreceptor topography," J. Comp. Neurol. 292, 497-523 (1990).

18. A. W. Snyder and M. Hamer, "The light-capture area of a photoreceptor," Vision Res. 12, 1749-1753 (1972).

19. W. S. Stiles and B. H. Crawford, "The luminous efficiency of rays entering the eye pupil at different points," Proc. R. Soc., London 112, 428-450 (1933).

20. M. J. Piket-May, A. Taflove, and J. B. Troy, "Electrodynamics of visible-light interactions with the vertebrate retinal rod," Opt. Lett. 18, 568-570 (1993).

21. N. P. A. Zagers and D. van Norren, "Absorption of the eye lens and macular pigment derived from the reflectance of cone photoreceptors," J. Opt. Soc. Am. A 21, 2257-2268 (2004).

22. J. M. Gorrand and F. C. Delori, "Reflectance and curvature of the inner limiting membrane at the foveola," J. Opt. Soc. Am. A 16, 1229-1237 (1999).

23. J. van de Kraats and D. van Norren, "Optical density of the aging human ocular media in the visible and the UV," J. Opt. Soc. Am. A 24, 1842-1857 (2007)

24. R. C. Smith and K. S. Baker, "Optical properties of the clearest natural waters," Appl. Opt. 20, 177-184 (1981).

25. G. J. Handelman, D. M. Snodderly, N. I. Krinsky, M. D. Russett, and A. J. Adler, "Biological control of primate macular pigment. Biochemical and densitometric studies," Invest. Ophthalmol. Visual Sci. 32, 257-267 (1991).

26. O. W. van Assendelf, in Spectroscopy of Hemoglobin Derivatives, Royal Van Gorcum Ltd., Assen, The Netherlands (1970). 
27. V. P. Gabel, R. Birngruber, and F. Hillenkamp, "Visible and near infrared light absorption in pigment epithelium and choroid," in Excerpta Medica, International Congress Series, K. Shimizu and J. A. Oosterhuis, Eds., pp. 658-662, Elsevier, Amsterdam (1978).

28. "Guidelines on limits of exposure to broad-band incoherent optical radiation (0.38 to 3 microM)," International Commission on NonIonizing Radiation Protection, Health Phys. 73, 539-554 (1997).

29. T. T. J. M. Berendschot and D. van Norren, "On the age dependency of the macular pigment optical density," Exp. Eye Res. 81, 602-609 (2005).

30. W. H. Press, B. P. Flannery, S. A. Teukolsky, and W. T. Vetterling, Numerical Recipes in Pascal, Cambridge University Press, Cambridge (1989).

31. J. T. P. van den Berg and K. E. Tan, "Light transmittance of the human cornea from 320 to $700 \mathrm{~nm}$ for different ages," Vision Res. 34, 1453-1456 (1994)

32. J. J. Vos, J. Walraven, and A. van Meeteren, "Light profiles of the foveal image of a point source," Vision Res. 16, 215-219 (1976).

33. R. A. Bone, J. T. Landrum, L. Fernandez, and S. L. Tarsis, "Analysis of the macular pigment by HPLC: retinal distribution and age study," Invest. Ophthalmol. Visual Sci. 29, 843-849 (1988).

34. W. M. R. Broekmans, T. J. M. Berendschot, W. A. Klöpping, A. J. de Vries, R. A. Goldbohm, C. C. Tijssen, M. Karplus, and G. van Poppel, "Macular pigment density in relation to serum and adipose tissue concentrations of lutein and serum concentrations of zeaxanthin," Am. J. Clin. Nutr. 76, 595-603 (2002).

35. J. M. Nolan, J. Stack, O. O'Donovan, E. Loane, and S. Beatty, "Risk factors for age-related maculopathy are associated with a relative lack of macular pigment," Exp. Eye Res. 84, 61-74 (2007).

36. J. van de Kraats, T. T. Berendschot, S. Valen, and D. van Norren, "Fast assessment of the central macular pigment density with natural pupil using the macular pigment reflectometer," J. Biomed. Opt. 11, 064031 (2006)

37. I. Lengyel, A. Tufai, H. A. Hosaini, P. Luthert, A. C. Bird, and G. Jeffery, "Association of drusen deposition with choroidal intercapillary pillars in the aging human eye," Invest. Ophthalmol. Visual Sci. 45, 2886-2892 (2004).

38. K. U. Loffler and W. R. Lee, "Basal linear deposit in the human macula," Graefe's Arch. Clin. Exp. Ophthalmol. 224, 493-501 (1986)
39. L. Feeney-Burns and M. R. Ellersieck, "Age-related changes in the ultrastructure of Bruch's membrane," Am. J. Ophthalmol. 100, 686697 (1985).

40. J. E. Grunwald, S. M. Hariprasad, and J. DuPont, "Effect of aging on foveolar choroidal circulation," Arch. Ophthalmol. (Chicago) 116, 150-154 (1998).

41. R. S. Ramrattan, T. L. van der Schaft, C. M. Mooy, W. C. de Bruijn, P. G. H. Mulder, and P. T. V. M. de Jong, "Morphometric analysis of Bruchs membrane, the choriocapillaris, and the choroid in aging," Invest. Ophthalmol. Visual Sci. 35, 2857-2864 (1994).

42. L. Feeney-Burns, E. S. Hilderbrand, and S. Eldridge, "Aging human RPE: morphometric analysis of macular, equatorial, and peripheral cells," Invest. Ophthalmol. Visual Sci. 25, 195-200 (1984).

43. T. Sarna, J. M. Burk, W. Korytowski, M. Rozanowska, C. M. Skumatz, A. Zareba, and M. Zareba, "Loss of melanin from human RPE with aging: possible role of melanin photooxidation," Exp. Eye Res. 76, 89-98 (2003).

44. J. J. Weiter, F. C. Delori, G. L. Wing, and K. A. Fitch, "Retinal pigment epithelial lipofuscin and melanin and choroidal melanin in human eyes," Invest. Ophthalmol. Visual Sci. 27, 145-152 (1986).

45. M. Boulton, F. Docchio, P. Dayhaw-Barker, R. Ramponi, and R. Cubeddu, "Age-related changes in the morphology, absorption, and fluorescence of melanosomes and lipofuscin granules of the retinal pigment epithelium," Vision Res. 30, 1291-1303 (1990).

46. N. P. Zagers and D. van Norren, "Photoreceptors act as spectrally flat reflectors," Invest. Ophthalmol. Visual Sci. 44, ARVO abstract No. 2873 (2003).

47. S. S. Choi, N. Doble, J. Lin, J. Christou, and D. R. Williams, "Effect of wavelength on in vivo images of the human cone mosaic," J. Opt. Soc. Am. A 22, 2598-2605 (2005).

48. S. A. Burns, S. Wu, J. C. He, and A. E. Elsner, "Variations in photoreceptor directionally across the central retina," J. Opt. Soc. Am. A 14, 2033-2040 (1997).

49. B. Vohnsen, I. Iglesias, and P. Artal, "Guided light and diffraction model of human-eye photoreceptors," J. Opt. Soc. Am. A 22, 2318 2328 (2005)

50. C. A. Curcio, C. L. Millican, K. A. Allen, and R. E. Kalina, "Aging of the human photoreceptor mosaic: evidence for selective vulnerability of rods in central retina," Invest. Ophthalmol. Visual Sci. 34 3278-3296 (1993) 